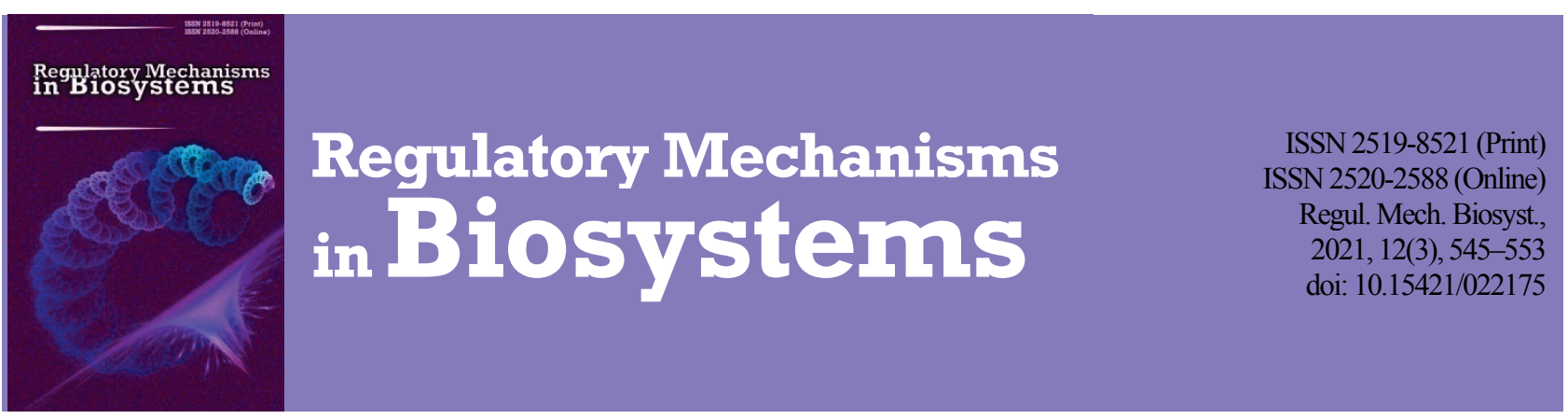

\title{
Course correction of adjuvant arthritis with cryopreserved multipotent mesenchymal stromal cells
}

\author{
D. B. Vvedenskyi****, N. O. Volkova*, M. S. Yukhta*, N. O. Ashukina***, A. M. Goltsev* \\ *Institute for Problems of Cryobiology and Cryomedicine of the National Academy of Sciences of Ukraine, Kharkiv, Ukraine \\ **V. N. Karazin Kharkiv National University, Kharkiv, Ukraine \\ ***Sytenko Institute of Spine and Joint Pathology National Academy of Medical Sciences of Ukraine
}

Article info

Received 03.07.2021

Received in revised form 05.08.2021

Accepted 06.08.2021

Institute for Problems of Cryobiology and Cryomedicine of the National Academy of Sciences of Ukraine, Pereyaslavskaya st., 23 Kharkiv, 61016, Ukraine Tel.: +38-066-129-85-97. E-mail:volkovana781@gmail.com

V. N. Karazin Kharkiv National University, Svobodysq., 4. Kharkiv, 61022, Ukraine Tel.: +38-063-875-44-09. E-mail:vvedenskyd@ukr.net

Sytenko Institute of Spine and Joint Pathology National Academy of Medical Sciences of Ukraine, Pushkinskaya st., 80, Kharkiv, 61024, Ukraine. Tel.: +38-057-725-14-51. E-mail: nataliya_ashukina@gmail.com
Vvedenskyi, D. B., Volkova, N. O., Yukhta, M. S., Ashukina, N. O., \& Goltsev, A. M. (2021). Course correction of adjuvant arthritis with cryopreserved multipotent mesenchymal stromal cells. Regulatory Mechanisms in Biosystems, 12(3), 545-553. doi:10.15421/022175

Rheumatoid arthritis is an inflammatory autoimmune disease that occurs as a result of impaired immune tolerance, leading to an aberrant immune response to autologous antigens. Multipotent mesenchymal stromal cells (MMSCs) and the biologically active substances they produce can promote the activation of regenerative processes in the organism not only by direct cell differentiation, but also due to their inherent trophic and immunosuppressive potentials. The aim of the study was to experimentally evaluate changes in the course of the acute phase of adjuvant arthritis upon local and generalized administration of cryopreserved MMSCs from adipose and cartilage tissues. The results of histological, imunohistochemical and biochemical studies showed that the animals of the control group throughout the observation period developed an inflammatory process, which manifested in joint swelling (increased arthritis index), leukocytosis, spread of chondrocyte-free zones, weakening of staining, loss of clarity of cartilage tissue contours, increased content of cyclooxygenase-2, reduced glycosaminoglycan content and total antioxidant defense system activity. At the same time, the local administration of cryopreserved MMSCs from adipose and cartilage tissues contributed to the normalization of the structural and functional organization, content of glycosaminoglycans and cyclooxygenase- 2 with complete recovery of blood parameters. Less pronounced regeneration processes in articular cartilage occurred under generalized administration of cryopreserved MMSCs from adipose and cartilage tissues in comparison with the local method. However, the difference between the control and experimental groups indicates the ability of cryopreserved MMSCs to influence the intensity of regenerative processes in damaged cartilage tissue with both methods of administration. Comparative evaluation of the use of cryopreserved MMSCs from adipose and cartilage tissues showed the absence of significant changes in the studied indicators. These data can be used to substantiate and develop methods of arthritis treatment in clinical practice.

Keywords: cell therapy; cartilage; adipose tissue; method of administration; rats.

\section{Introduction}

The problem of rheumatic diseases management is relevant worldwide. According to the World Health Organization, in the developed countries of the world inflammatory diseases of the joints and connective tissue occupy the second place after cardiovascular pathology among the causes of disability and mortality. Rheumatoid arthritis is characterized by autoimmune chronic inflammatory synovitis with progressive joint destruction and a wide range of extra-articular (systemic) manifestations (Rana et al., 2018). Currently, nonsteroidal anti-inflammatory drugs, analgesics, glucocorticoids, synthetic basic antiinflammatory drugs and genetically engineered biological drugs are used in the treatment of this pathology, differentiated into five classes: tumor necrosis factor inhibitors; antiB-cell drugs; T-cell costimulation blocker, 1- and 6-interleukin receptor antagonists (Burmester \& Pope, 2017; Bullock et al., 2018; Goryachev \& Telnykh, 2018). Intra-articular injections are also widely used in rheumatoid arthritis in addition to systemic drugs. For example, topical glucocorticoid therapy reduces inflammatory activity in joint tissues by reducing cellular infiltration and expression of proinflammatory cytokines that induce synovitis (Savvidou et al., 2019). And local administration of tumor necrosis factor inhibitors leads to a significant reduction in the proliferation of synovial tissue cells (Zhang \& Ma, 2018). Despite of the presence of a large number of antirheumatoid drugs on the world pharmaceutical market, as well as certain successes in the development of various protocols for their combined application, rheumatoid arthritis therapy remains a relevant task. Many factors are engaged in initiation and maintenance of the autoimmune process in patients with rheumatoid arthritis (Chen et al., 2018). One of possible causes of the disease is disregulation of signal systems providing the balance between the processes of apoptosis and proliferation. Actually, in multicellular organisms apoptosis acts as an integral component. Its genetic and metabolic impairments contribute greatly to pathogenesis of some pathologies, including rheumatoid arthritis (Phull et al., 2018). Against this background there was noted an infiltration of synovial membrane with lymphocytes, monocytes, plasmatic cells, which under co-operative interaction release the mediators, provoking an inflammatory reaction and destruction of tissues.

The main goal of rheumatoid arthritis treatment is to achieve complete or partial reduction of disease activity. Previously, the position was established that treatment with antirheumatic drugs was "good enough", and recovery was considered as unattainable. Today, it is believed that basic therapy can slow the progression of the disease and induce permanent remission, especially if it is started with duration of symptoms less than 12 weeks. If their application is delayed, then not all basic drugs can "have time" to show their effect. The longer the duration of the disease (long-term suffering from rheumatoid arthritis), the worse the response to the "basic" drugs in comparison with patients receiving this therapy at an early stage of the disease. It is better if they are prescribed immediately after the diagnosis is made. Based on the analogy with basic therapy, in 
our study we chose the early phase of experimental adjuvant arthritis to assess the effectiveness of cell therapy (Kim, 2016).

Unfortunately, traditional treatment approaches, influencing only certain links in the pathogenesis of rheumatoid arthritis, can be effective in arresting the development of a pathological process in the joints, but their use has no restorative effect. Another problem in the treatment of autoimmune diseases is that the immune system is complex, and the applied drugs are usually targeted at only one or a small subset of the system (Conigliaro et al., 2019). In addition, some pharmacopoeia drugs used in the treatment of arthritis are addictive and have side effects (Ingawale \& Patel, 2018).

However, regenerative processes in affected joints with rheumatoid arthritis can be stimulated by multipotent mesenchymal stromal cells (MMSCs). Today, the isolation, cultivation and cryopreservation of the cells with mesenchymal origin is the basis for obtaining cellular material, which is used in regenerative medicine for the treatment of pathologies of various genesis (Fitzsimmons et al., 2018). It is known that MMSCs can be isolated from various tissues such as bone marrow, fat, cartilage and some others (Li et al., 2018; Sasaki et al., 2019; Neybecker et al., 2020). Depending on the source, the properties of MMSCs differ slightly. For example, MMSCs from adipose tissue have a higher level of cytokine secretion compared to bone-marrow derived MMSCs (To \& Khan, 2019). So, depending on the type of cells selected to restore the tissue architecture, the influence of growth factors can vary (Freyria \& MalleinGerin, 2012). As for cartilage stem cells, they have been reviewed in a few in vivo and in vitro studies to date. The proliferative potential and ability for multilinear differentiation of progenitor cells isolated from hyaline cartilage have been studied (Levato et al., 2017). This observation has significantly improved our understanding the pathophysiology of this tissue, and has also contributed to the search for a new potential tool for the treatment of its damage. Several research groups have shown that cells derived from cartilage also meet MMSC criteria. They have high proliferative and clonogenic potentials, multipotent properties and phenotype inherent in MMSCs. These cells are called "cartilage derived stromal cells", "dedifferentiated chondrocytes", and "articular-derived dedifferentiated chondrocytes" in the literature (Peng et al., 2008; Freitag et al., 2016).

Today, MMSCs remain the subject of special attention because they are characterized by multipotency, trophic and immunomodulatory potentials, they are able to maintain hematopoiesis and produce cytokines, chemokines and growth factors (Fan et al., 2020). In general, such properties make MMSCs one of the main candidates for biotechnological and tissue engineering developments. And because these cells can involve complex mechanisms that the body naturally uses to modulate and repair immune imbalances, MMSC therapy has the potential to treat and correct autoimmune disorders, including rheumatoid arthritis, in ways that conventional drugs cannot yet engage. Many studies are currently being conducted in this area, but in the works on MMSCs obtained from various sources as well as very different treatment, different dosing regimens were used. In particular, the timing of cell therapy and the method of administration of MMSCs remain unregulated (To \& Khan, 2019).

We have previously shown that cell cultures derived from bone marrow and alternative sources (tendon, adipose and cartilage) have the phenotype of progenetor cells of mesenchymal origin, retain the ability for proliferation, colony formation and migration. Cryopreserved MMSCs (CrMMSC) from these investigated sources kept membrane integrity, ability to adhesion, colony formation, proliferation, synthesis of type I collagen and directed differentiation. The analysis of phenotype showed that the tested CrMMSCs culture was characterized by high levels of expression ( $\geq 90 \%$ ) of CD44, CD90, CD105, CD73 and low expression $(\leq 1 \%)$ of hematopoietic marker CD45. Cell cultures derived from all studied sources have phenotype of precursor cells of mesenchymal origin. The MMSCs of cartilage tissue are characterized by a greater capacity for colony formation and proliferation, and lower capacity for directed differentiation than MMSCs from bone marrow and adipose tissue (Volkova et al., 2016). In another previous study (Volkova et al., 2019) we investigated the effect of growth and differentiation factors on CrMMSCs from bone marrow, adipose and cartilage tissues. It was shown that the addition of fibroblast growth factor to the culture medium resulted in an increase of proliferative potential of CrMMSCs from bone marrow and adipose tissues. And the use of transforming growth factor $\beta$ increased the synthesis of type II collagen and glycosaminoglycans by these cells during the culturing. But response of MMSCs from bone marrow to the influence of these growth factors was more pronounced in comparison with adiposederived ones. Proliferative activity, the synthesis of type II collagen and glycosaminoglycans of CrMMSCs from cartilage tissue did not undergo changes under the influence of the investigated factors, but they were characterized by a relatively greater activity of synthetic processes compared to CrMMSCs from bone marrow and adipose tissues.

Modeling of the course of rheumatoid arthritis phases is a complex multifactorial task. And the use of cell therapy by local and generalized administration involves the correction of both damaged joint and immune status of the recipient.

The aim of the study was to experimentally evaluate changes in the course of acute phase of adjuvant arthritis upon local and generalized administration of cryopreserved adipose- and cartilage-derived mesenchymal stromal cells.

\section{Materials and methods}

All animal experiments were performed according to the international principles of bioethics, laws of Ukraine, materials of the IV European Convention for the Protection of Vertebrate Animals Used for Experimental and Other Scientific Purposes (March 18, 1986), and the protocol of the Bioethics Committee of Institute for Problems of Cryobiology and Cryomedicine of the National Academy of Sciences of Ukraine (No. 2018-01). Outbreed white male rats (aged 12-14 weeks, $n=35$ ) were used in the study. The rats were kept in plastic cages (five animals each) at a controlled temperature $\left(18-22{ }^{\circ} \mathrm{C}\right)$, humidity $(30-70 \%)$ and lighting (light interval from $8^{00}$ to $20^{00}$ ) with free access to water and food according to the standard diet.

Primary suspension of cells from biopsies of adipose and cartilage tissues of rats $(n=5)$ was obtained by enzymatic digestion (Volkova et al., 2016). To do this, tissue samples were washed with Hanks' solution (PAA, Austria) with gentamicin $(150 \mu \mathrm{g} / \mathrm{mL})$ (Farmak, Ukraine) and incubated in a type II collagenase solution $(1.5 \mathrm{mg} / \mathrm{mL}$ ) (PanEco, Russia) at $4{ }^{\circ} \mathrm{C}$ for 18 hours. Then cells were isolated from biopsies by resuspension followed by centrifugation at $1500 \mathrm{rpm}$ for 3 minutes. Supernatant was removed, culture medium was added to the precipitate and the cell suspension was placed on culture plastic. The seeding density of the cells was $1 \cdot 10^{4}$ per $1 \mathrm{~cm}^{2}$ of culture flasks. The culture medium in all cases contained: IMDM medium (RAA, Austria), 10\% fetal bovine serum (HyClone, USA), kanamycin (150 $\mu \mathrm{g} / \mathrm{mL})$ (Farmak, Ukraine) and amphotericin B $(5 \mu \mathrm{g} / \mathrm{mL})$ (RAA, Austria). The culture medium was changed every three days. The standard culturing conditions at $37{ }^{\circ} \mathrm{C}$ in an atmosphere of $5 \% \mathrm{CO}_{2}$ in an incubator (Sanyo, Japan) were used. Upon reaching a monolayer, cell cultures were subcultured using a $0.25 \%$ solution of trypsin (PAA, Austria) and versene (PanEco, Russia) in a ratio of 1:1.

Cryopreservation of MMSC cultures was carried out under the protection of $10 \%$ dimethyl sulfoxide (PanEco, Russia) with addition of $20 \%$ fetal bovine serum. The cryoprotectant solution was prepared on the culture medium. The resulting suspension was placed in Nunc CryoTubes (Sigma-Aldrich, USA) to $1 \mathrm{~mL}$. The cooling rate was $1{ }^{\circ} \mathrm{C} / \mathrm{min}$ to $-80^{\circ} \mathrm{C}$ followed by immersion in liquid nitrogen (Volkova \& Goltsev, 2015). Warming was carried out in a water bath at $40{ }^{\circ} \mathrm{C}$ until the liquid phase. Removal of the cryoprotectant was performed by adding Hanks' solution 1:9 followed by centrifugation at $1500 \mathrm{rpm}$ for 5 minutes.

After cryopreservation, the number of cells in the samples was counted using a Goryaev chamber. Metabolic activity of the cells was determined using the MTT assay. For this, $0.5 \mathrm{~mL}$ of MTT solution (Sigma, USA) at a concentration of $5 \mathrm{mg} / \mathrm{mL}$ was added to the test samples $\left(1 \cdot 10^{6}\right.$ cells $)$ and incubated for three hours at $37^{\circ} \mathrm{C}$. Then the medium was completely removed and $1 \mathrm{~mL}$ dimethyl sulfoxide per sample was added to dissolve the formazan crystals with following centrifugation for $10 \mathrm{~min}$ at $1000 \mathrm{rpm}$. The optical density of formazan solution in the supernatant was measured using a biochemical analyzer CHEM 7 (ERBA, Czech Republic) at a wavelength of $540 \mathrm{~nm}$. Cell-free medium was used as a blank. Morphological characteristics of CrMMSCs were examined on day 10 of culturing. Cell preparations were fixed with $4 \%$ paraformalde- 
hyde solution, followed by azure-eosin staining by Romanowsky-Giemsa for $10 \mathrm{~min}$ at room temperature.

The adjuvant arthritis model was induced in male rats by subplantar administration of complete Freund's adjuvant at a dose of $0.25 \mathrm{~mL}$ (Santa Cruz, USA). The chosen experimental model is adequate to rheumatoid arthritis changes in humans: adjuvant induced arthritis shares common symptoms like joint swelling, lymphocyte infiltration and cartilage degradation (Choudhary, 2018). CrMMSCs from adipose and cartilage tissues of rats was used in the work. The scheme of the experiment is shown in Figure 1.

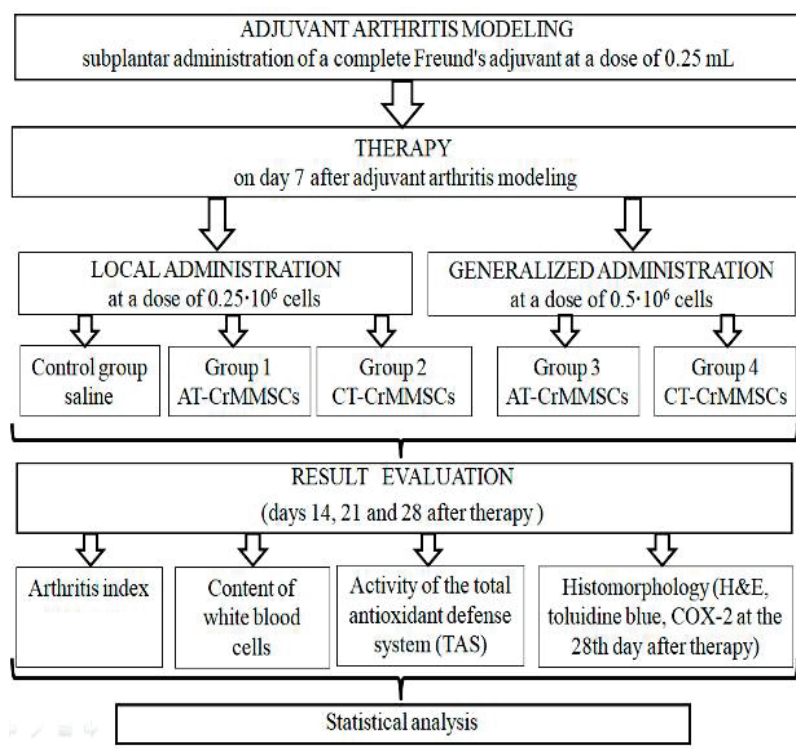

Fig. 1. Experimental scheme: AT-CrMMSCs - cryopreserved mesenchymal stromal cells from adipose tissue; CT-CrMMSCs cryopreserved mesenchymal stromal cells from cartilage tissue

On the 7th day of adjuvant arthritis modeling, experimental animals were administered: control group $(\mathrm{n}=5)$ - saline; experimental group 1 $(n=5)-$ CrMMSCs from adipose tissue at a dose of $0.25 \cdot 10^{6}$ cells locally; experimental group $2(\mathrm{n}=5)-\mathrm{CrMMSCs}$ from cartilage tissue at a dose of $0.25 \cdot 10^{6}$ cells locally; experimental group $3(n=5)-$ CrMMSCs from adipose tissue at a dose of $0.5 \cdot 10^{6}$ cells generalized; experimental group $4(n=5)-$ CrMMSCs from cartilage tissue at a dose of $0.5 \cdot 10^{6}$ cells generalized. A group of intact animals $(n=5)$ was also formed as a control. On days 14, 21, and 28, arthritis index, white blood cell count, and serum total antioxidant defense system (TAS) activity were determined. The arthritis index was defined as a ratio of the joint circumference of the experimental paw to the undamaged joint of the same animal. TAS activity was estimated quantitatively by the method of UV spectrophotometry (ERBA CHEM 7, Erba Lachema sro, Brno, Czech Republic) using test kit (Prod. No. NX2332, Randox Laboratories Ltd., UK) according to the manufacturer's instructions and normalized to $1 \mathrm{mg}$ of protein (Prod. No. TP8336, Randox Laboratories Ltd., UK).

At the 28th day after therapy, the animals of the control and experimental groups were taken out from the experiment. Humane euthanasia by $\mathrm{CO}_{2}$ asphyxiation was performed. The ankle joints of the rats were isolated for histological examination. The material was fixed in a $10 \%$ solution of neutral formalin, decalcified in a $4 \%$ solution of formic acid, dehydrated in ethyl alcohols of increasing concentrations (from $70 \%$ to $96 \%$ ), in a solution of $96 \%$ ethanol with chloroform (1:1) with subsequent embedding material in paraffin (Dey, 2018). Axial histological sections of the joints were made on the Reichert sledge microtome (Austria) and stained with Weigert's hematoxylin and eosin. ZEISS Primo Star light microscope (Carl Zeiss Microscopy GmbH, Germany) was used for the analysis of histological preparations. An assessment of histological changes in cartilage tissue was carried out according to OARSI scale adapted for rats (Gerwin et al., 2010). Histochemical staining with toluidine blue (Flyka, Germany) was performed on deparaffinized sections of joints. The relative area of the sites with staining to glycosaminoglycans (purple) was measured using the program ZEISS ZEN 2 (blue edition) (Carl Zeiss
Microscopy GmbH, Germany) and the percentage was determined as the ratio of the area of metachromatic staining to the total area of articular cartilage taken as $100 \%$.

Immunohistochemical staining of deparaffinized sections of the ankle joints was performed using monoclonal antibodies to cyclooxygenase-2 (COX-2) at a titer of 1:100 (Thermo Fisher Scientific, USA). Staining was performed according to the protocol recommended for the peroxidase detection system Ultra Vision Quantro HRP DAB (Thermo Fisher Scientific, USA). COX-2 positively stained cells (brown) were counted using the ZEISS ZEN 2 (blue edition) program (Carl Zeiss Microscopy GmbH, Germany), and their percentage was determined as the ratio of the number of stained cells to the total number of cells (per $1 \mathrm{~mm}^{2}$ ) taken as $100 \%$. For negative control of COX-2, deparaffinized sections were stained according to the same protocol, replacing the antibodies with the mouse immunoglobulin $\mathrm{G}$ isotype.

The significance of the differences was assessed by means of a Kruskal-Wallis one-way analysis of variance on ranks, followed by StudentNewman-Keuls multiple comparison tests for independent samples and using the Friedman test with the Newman-Keuls method for aposterior comparisons for dependent samples at a significance level of $\mathrm{P}<0.05$ with the program Statistika 8 (StatSoft Inc., USA).

\section{Results}

The results of the MTT-test showed that CrMMSCs from cartilage tissue were more metabolically active by 1.50 times than adipose derived cells (Fig. 2a). Under culturing conditions CrMMSCs from adipose tissue were characterized by the presence of sail-, star- and spindle-shaped cellular elements (Fig. 2b). CrMMSCs from cartilage tissue were represented by polygonal and spindle-shaped cells (Fig. 2c).

Evaluation of general condition showed that the animals with saline administration (control group) had signs of inflammation in the joint edema and hyperemia as well as an increase in the arthritis index by 1.58 times relative to the intact animals at the 14th day of modeling and this tendency continued throughout the experiment. In animals of experimental groups 1 and 2, severity of joint edema at the 14th day was 1.15and 1.20-fold decreased, respectively, compared to the control group. At the 28th day of observation in animals of theses groups the arthritis index was respectively 1.26 - and 1.28 -fold lower than in the group with saline administration. Generalized administration led to less pronounced decrease in this index compared to local one but the downward trend persisted. However, the arthritis index did not reach the level of intact animals in any studied group (Table 1).

The serum overall activity of the antioxidant system in animals with saline administration was significantly reduced relative to intact rats throughout the observation period. It should be noted that in animals of all experimental groups, the studied indicator tended to normalize from the 21 st day. Thus, at the 28th day, the studied indicator in groups 1 and 2 was higher by 1.92 and 2.12 times, respectively, in relation to the control group. The TAS activity in groups 3 and 4 on day 28 was 1.67 - and 1.60-fold higher, respectively, compared to the group of animals with saline administration. Therefore, recovery of the antioxidant defense system was more intense in the group of animals with local administration of CrMMSCs from cartilage tissue (Table 2). As is known, indices of peripheral blood are therefore important not only for diagnostic and prognostic criteria of the course of rheumatoid arthritis, but also as a reflection of treatment effectiveness. In our study the number of leukocytes in animals with saline administration was increased throughout the experiment. In animals with local therapy, the dynamics of white blood cell count tended to decrease from the 14th day of observation. And at day 28, the studied indicator in groups 1 and 2 did not statistically differ from intact animals. The content of leukocytes in groups 3 and 4 on day 28 after therapy was respectively 1.22- and 1.19-fold lower compared to the group of animals with saline administration, but normalization of this parameter in contrast to the first two experimental groups did not occur (Table 3 ).

The micrographs of histological sections represented in Figure 3 showed that while the ankle joint of the intact animals was normal, rough structural abnormalities were found in the joints of the control animals (saline administration). In this group cartilage was absent at most of the 
articular ends of the bones, and the remaining islands of fibrous cartilage had acellular eosinophilic areas, which corresponded to 4-5 degrees of lesion according to the OARSI scale adapted for rats.

In animals with adjuvant arthritis and local administration of CrMMSCs from adipose and cartilage tissues, histological examination showed minor destructive changes in joint cartilage (1-2 degree of damage according to the adapted OARSI scale), subchondral bone, synovial membrane and joint capsule. On the articular surface of both the tibia and heel bones, articular cartilage was found, the surface area of which was uneven, slightly fibrous, with eosinophilic staining of the matrix. Elongated chondrocytes with oval hyperchromic nuclei, a narrow rim of the cytoplasm and the orientation of the long axis parallel to the articular surface were localized in several layers. Chondrocytes in the necrobiosis state were found in the intermediate zone of the cartilage. Phenomena of cell order disturbance, empty lacunae, uneven staining of the matrix were also revealed in the case of adipose-derived CrMMSC administration. The area of calcified cartilage had a characteristic structure in both experimental groups.
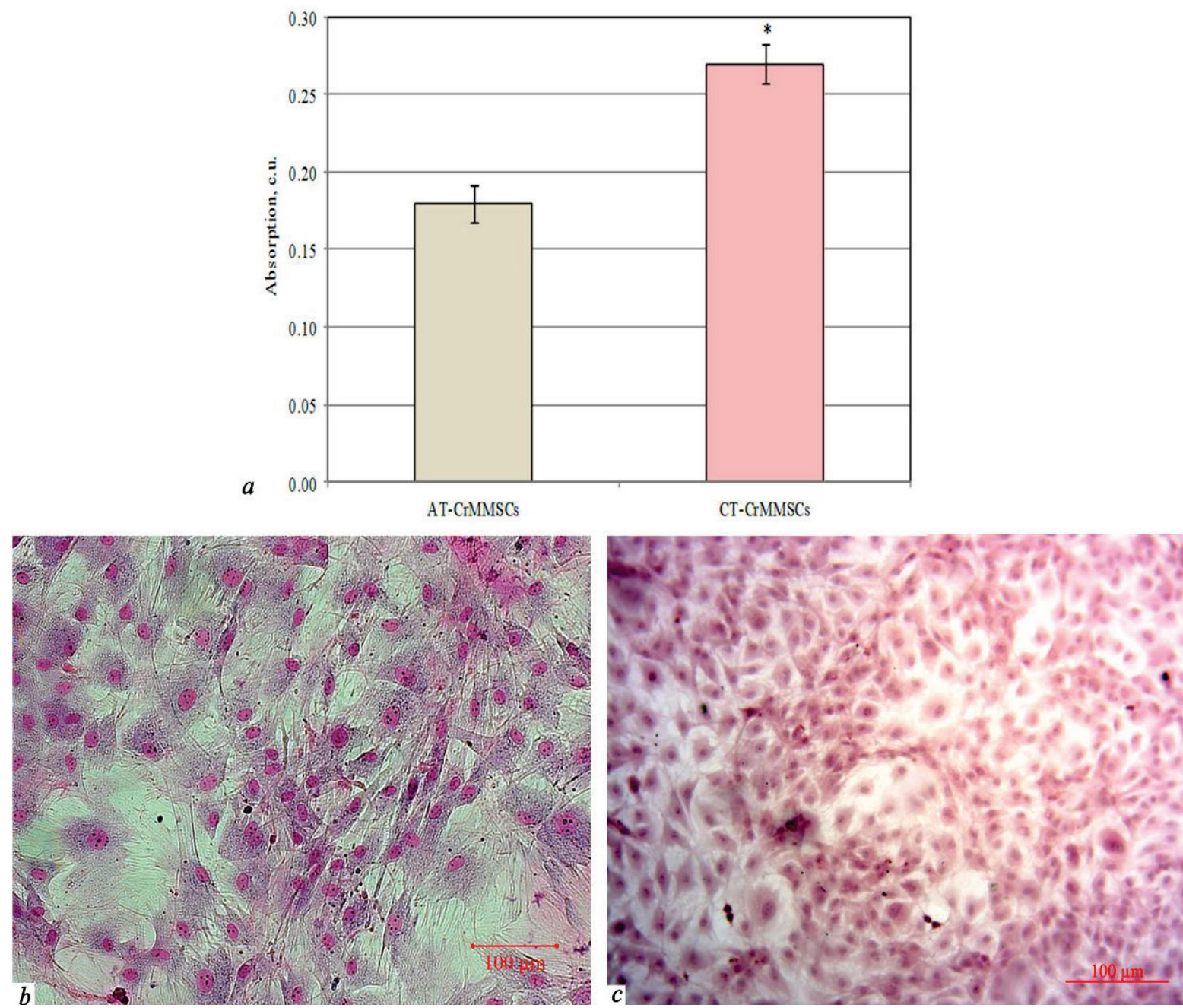

Fig. 2. Proliferative and morphological characteristics of CrMMSCs from adipose and cartilage tissues: $a$-MTT-test; $b$-micrograph of CrMMSCs from adipose tissue on the 10th day of culturing; $c$ - micrograph of CrMMSCs from cartilage tissue on the 10th day of culturing; light microscopy, azure-eosin staining; AT-CrMMSCs - cryopreserved mesenchymal stromal cells from adipose tissue; CT-CrMMSCs - cryopreserved mesenchymal stromal cells from cartilage tissue; *- the difference is statistically significant relative to cryopreserved mesenchymal stromal cells from adipose tissue $(\mathrm{P}<0.05 ; \mathrm{n}=5)$

Table 1

Effect of CrMMSCs from adipose and cartilage tissues on dynamic of arthritis index in rats with adjuvant arthritis $(x \pm S D ; n=5)$

\begin{tabular}{|c|c|c|c|}
\hline Group & Day 14 & Day 21 & Day 28 \\
\hline Intact & $1.02 \pm 0.085^{\mathrm{a}, \mathrm{k}}$ & $1.02 \pm 0.085^{\mathrm{ak}}$ & $1.02 \pm 0.085^{\mathrm{ak} k}$ \\
\hline Control (saline ac & $1.584 \pm 0.057^{\mathrm{b}, \mathrm{k}}$ & $1.517 \pm 0.072^{\mathrm{b} \mathrm{k} \mathrm{k}}$ & $\begin{array}{l}1.553 \pm \\
0.037^{b \mathrm{kk}}\end{array}$ \\
\hline 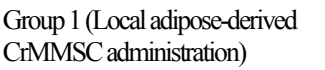 & $1.371 \pm 0.064^{\mathrm{ck}}$ & $1.263 \pm 0.079^{\text {c,dil }}$ & $0.031^{\mathrm{cll}}$ \\
\hline $\begin{array}{l}\text { Group } 2 \text { (Local cartilage } \\
\text { CrMMSC administratic }\end{array}$ & $1.323 \pm 0.097^{\mathrm{ck}}$ & $1.259 \pm 0.046^{\mathrm{cll}}$ & $1.21 / \pm$ \\
\hline $\begin{array}{l}\text { Group 3 (Generalized } \\
\text { derived CrMMSC ad }\end{array}$ & $1.450 \pm 0.030^{\mathrm{b}, \mathrm{k}}$ & $1.355 \pm 0.058^{\text {c,dl }}$ & $1.314 \pm 0.023^{\mathrm{dl}}$ \\
\hline $\begin{array}{l}\text { Group 4(Generalized cartilage- } \\
\text { derived CrMMSC administration) }\end{array}$ & $1.466 \pm 0.041^{\mathrm{b}, \mathrm{k}}$ & $1.382 \pm 0.037^{\mathrm{dl}}$ & $1.363 \pm 0.052^{\mathrm{dl}}$ \\
\hline
\end{tabular}

Note: ${ }^{\text {a, b, c, d }}$ indicate values that probably differed in one column of the table ( $\mathrm{P}<$ $0.05){ }^{\mathrm{k}, \mathrm{lm}}$ indicate values that probably differed in one row of the table $(\mathrm{P}<0.05)$.

\section{Table 2}

Effect of CrMMSCs from adipose and cartilage tissues on dynamic of total antioxidant defense system activity in rats with adjuvant arthritis $(\mathrm{x} \pm \mathrm{SD} ; \mathrm{n}=5)$

\begin{tabular}{|c|c|c|}
\hline Group & Day 14 & Day 21 \\
\hline Intact & $35.26 \pm 0.93^{\mathrm{a} ; \mathrm{k}}$ & $35.22 \pm 0.97^{\mathrm{a}: \mathrm{k}} 35.28 \pm 0.90^{\mathrm{a}, \mathrm{k} k}$ \\
\hline Control (saline administration) & $17.55 \pm 0.68^{\mathrm{b} ; \mathrm{k}}$ & $16.83 \pm 0.60^{\mathrm{bjk}} 16.46 \pm 0.32^{\mathrm{bjk} k}$ \\
\hline $\begin{array}{l}\text { Group } 1 \text { (Local adipose-derived } \\
\text { CrMMSC administration) }\end{array}$ & $22.24 \pm 0.77^{\mathrm{b}, \mathrm{k}}$ & $27.32 \pm 0.48^{\mathrm{c}, \mathrm{d} \mathrm{l} l} \mathrm{l} 31.59 \pm 0.27^{\mathrm{cm}}$ \\
\hline $\begin{array}{l}\text { Group } 2 \text { (Local cartilage-derived } \\
\text { CrMMSC administration) }\end{array}$ & $21.62 \pm 0.47^{\mathrm{b} ; \mathrm{k}}$ & $29.19 \pm 0.53^{\mathrm{cll}} 34.72 \pm 0.65^{\mathrm{cm}}$ \\
\hline $\begin{array}{l}\text { Group } 3 \text { (Generalized adipose- } \\
\text { derived CrMMSC administration) }\end{array}$ & $20.88 \pm 0.91^{\mathrm{b} ; \mathrm{k}}$ & $25.42 \pm 0.68^{\mathrm{c}, \mathrm{d}, \mathrm{l}} \mathrm{2} 27.55 \pm 0.58^{\mathrm{d}, \mathrm{m}}$ \\
\hline $\begin{array}{l}\text { Group } 4 \text { (Generalized cartilage- } \\
\text { derived CrMMSC administration) }\end{array}$ & $20.46 \pm 0.37^{\mathrm{b} ; \mathrm{k}}$ & $23.31 \pm 0.38^{\mathrm{dll}} 2$ \\
\hline
\end{tabular}

Note: see Table 1 
Table 3

Effect of CrMMSCs from adipose and cartilage tissues on white blood cell count in rats with adjuvant arthritis $(x \pm S D ; n=5)$

\begin{tabular}{|c|c|c|c|}
\hline Group & Day 14 & Day 21 & Day 28 \\
\hline Intact & $16.62 \pm 2.12^{\mathrm{a} ; \mathrm{k}}$ & $16.54 \pm 2.02^{\mathrm{a} \cdot \mathrm{k}}$ & $16.46 \pm 2.10^{\mathrm{a} . \mathrm{k} k}$ \\
\hline Control (saline administration) & $28.46 \pm 2.85^{\mathrm{b} ; \mathrm{k}}$ & $29.66 \pm 1.67^{\mathrm{bk} k}$ & $25.13 \pm 3.01^{\mathrm{bk}}$ \\
\hline $\begin{array}{l}\text { Group } 1 \text { (Local adipose-derived } \\
\text { CrMMSC administration) }\end{array}$ & $3.07^{\mathrm{ckk}}$ & $2.35^{\mathrm{ckl},}$ & qal! \\
\hline $\begin{array}{l}\text { Group } 2 \text { (Local cartilage-derived } \\
\text { CrMMSC administration) }\end{array}$ & $22.05 \pm 2.82^{\mathrm{ck}}$ & $21.08 \pm 1.95^{\mathrm{ckl}}$ & $18.15 \pm 1.36^{\mathrm{a}: \mathrm{l}}$ \\
\hline $\begin{array}{l}\text { Group } 3 \text { (Generalized adipose- } \\
\text { derived CrMMSC administration) }\end{array}$ & $26.51 \pm 2.19^{\mathrm{b}, \mathrm{k}}$ & $28.55 \pm 2.56^{\mathrm{bk}}$ & $\pm 1.70^{\mathrm{cl}}$ \\
\hline $\begin{array}{l}\text { Group } 4 \text { (Generalized cartilage- } \\
\text { derived CrMMSC administration) }\end{array}$ & $27.46 \pm 2.07^{\mathrm{bjk}}$ & $27.66 \pm 2.32^{\mathrm{bjk}}$ & $21.13 \pm 1.56^{\mathrm{cl}}$ \\
\hline
\end{tabular}

Note: see Table 1.
In the groups of animals with generalized administration of CrMMSCs the changes were found in articular cartilage (2-3 degree of damage according to the adapted OARSI scale), subchondral bone, synovial membrane and joint capsule, which reflect the chronicity of a moderate inflammation. Compared with local administration, a more pronounced lesion in the capsule was noted, which was manifested by a diffuse inflammatory infiltration. In the articular cartilage, the absence of the superficial zone, irregularity of chondrocytes arrangement and uneven staining of the extra cellular matrix in the middle zone were revealed. In addition, acellular areas, empty gaps, dystrophy and necrosis of chondrocytes were visualized. Additionally, in the group of animals with generalized administration of cartilage-derived CrMMSCs, a tidemark was usually not observed, and the zone of calcified cartilage was of different widths with sprouted vessels.
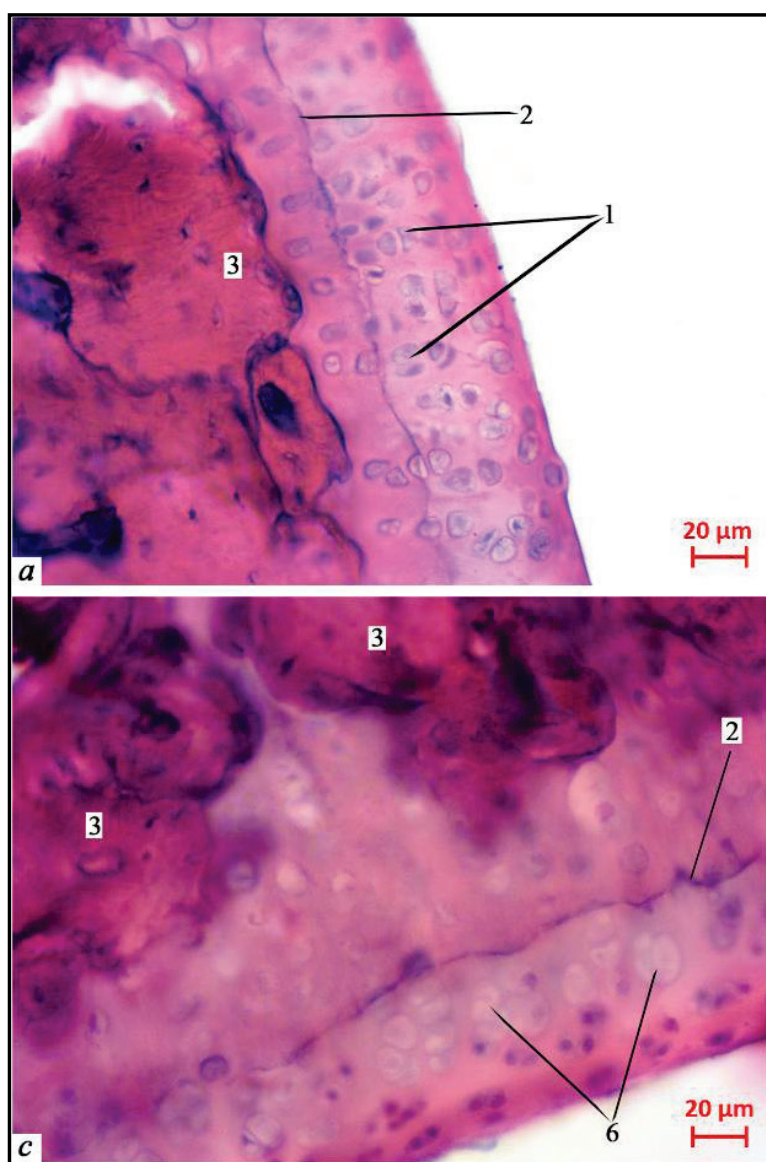

$c$

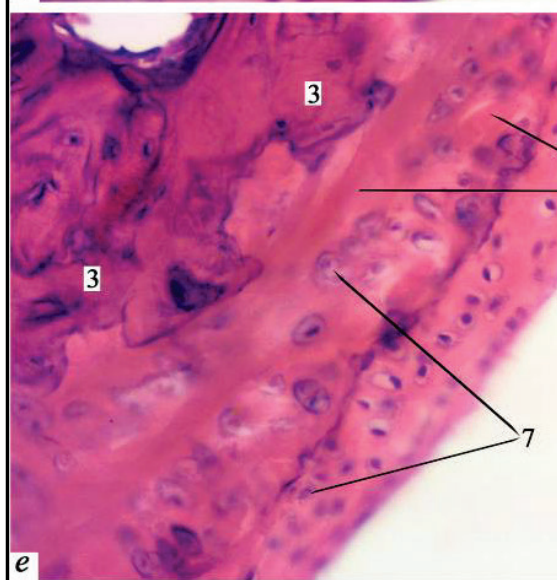

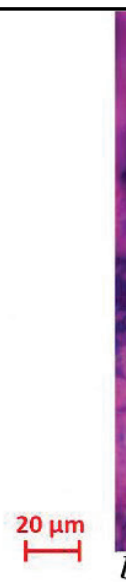

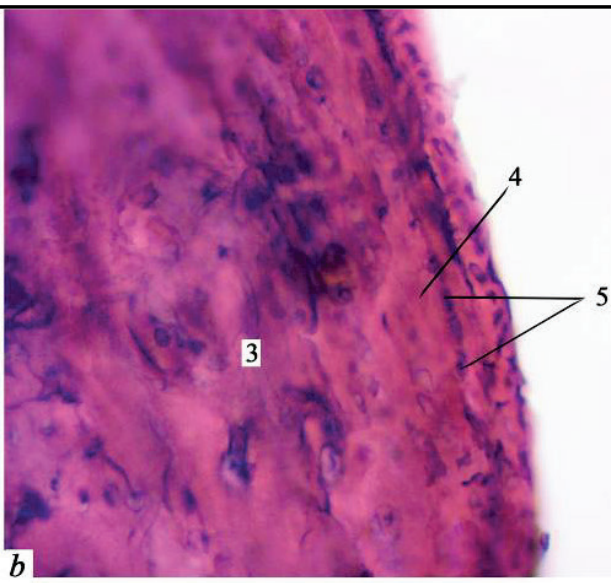

$20 \mu \mathrm{m}$
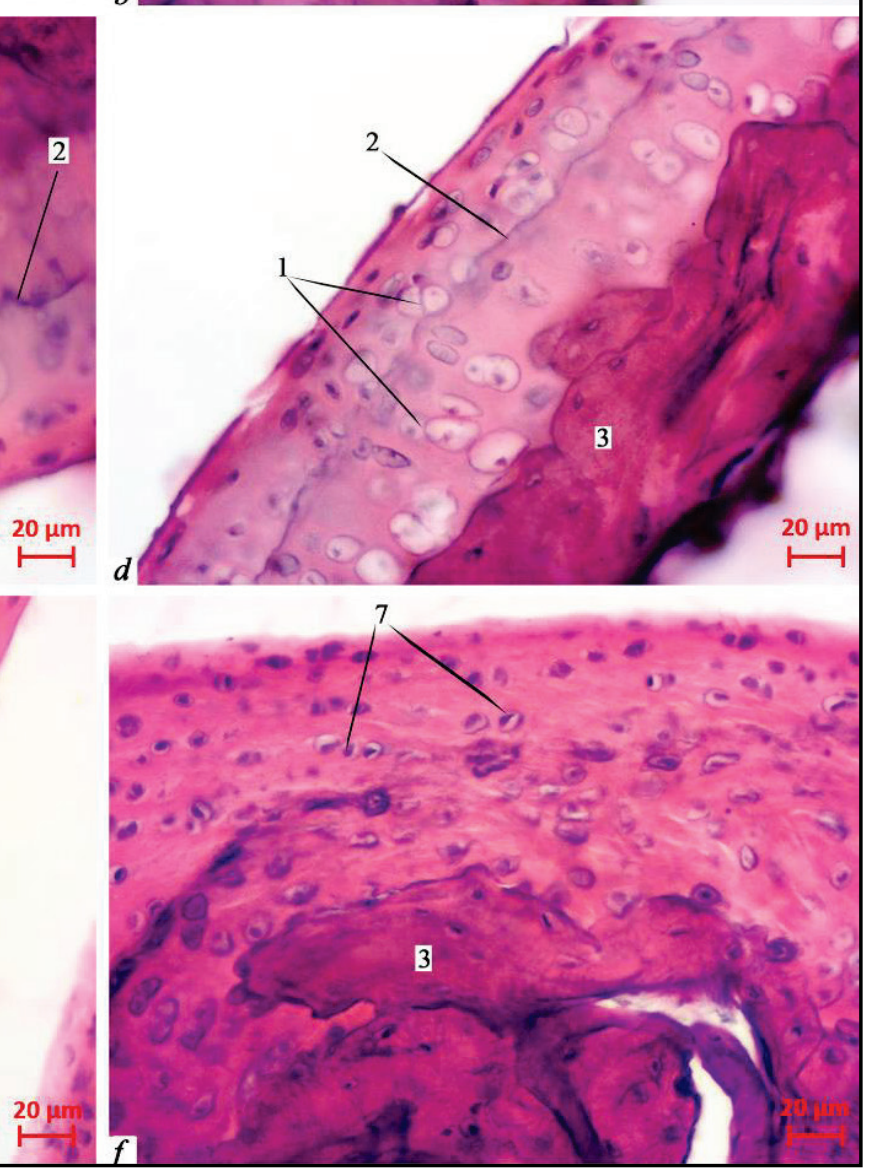

Fig. 3. Histological structure of articular cartilage of animals with adjuvant arthritis and CrMMSC therapy (28 day):

$a$-intact; $b$ - control (saline administration); $c$ - group 1 (local adiposed-derived CrMMSC administration); $d$ - group 2 (local cartilage-derived CrMMSC administration); $e$ - group 3 (generalized adiposed-derived CrMMSC administration); $f$-group 4 (generalized cartilage-derived CrMMSC administration); micrographs, light microscopy, hematoxylin and eosin staining; 1 - chondrocytes; 2 -tidemark; 3 -subchondral bone; 4 -acellular areas; 5 -fibrochondrocytes; 6 -empty lacunae; 7 -dystrophy of chondrocytes 
The next stage of the study was to investigate glycosaminoglycan content in articular cartilage of animals with adjuvant arthritis and therapy with CrMMSCs from adipose and cartilage tissues under local and generalized administration of the cells (Fig. 4). It is known that the one of characteristic signs of cartilage destruction is the loss of glycosaminoglycans by the matrix. Glycosaminoglycans are linear negatively charged heteropolysaccharides which are a mandatory component of the intercellular matrix and cell membranes. In the cartilage matrix, glycosaminoglycans are always bound to the protein and are part of the proteoglycans. The metabolism of proteoglycans and glycosaminoglycans changes at the earliest stages of articular cartilage damage. The loss of proteoglycans leads to defibering and cleavage of the matrix, changes in the processes of diffusion of metabolites in it, dehydration, disorganization and rupture of collagen fibers. In our study, the relative area of sites positively stained for glycosaminoglycans in the articular cartilage of intact animals was $92.6 \pm$ $5.6 \%$. In the control group of animals with saline administration, the studied index was $26.4 \pm 5.1 \%$, which is 3.51 -fold lower than intact one. In animals from groups 1 and 2, the relative area of sites positively stained for glycosaminoglycans was $61.7 \pm 5.7 \%$ and $69.3 \pm 6.1 \%$, which was respectively 2.34- and 2.63-fold higher than the corresponding values in the control group of animals. In animals with generalized administration (groups 3 and 4), the studied indicator was accordingly $48.7 \pm 5.2 \%$ and $45.1 \pm 6.8 \%$, which was 1.84 - and 1.71 -fold higher than the corresponding values in the group of animals with saline administration. However, it should be noted that in all studied groups, the glycosaminoglycan content did not reach the intact value.

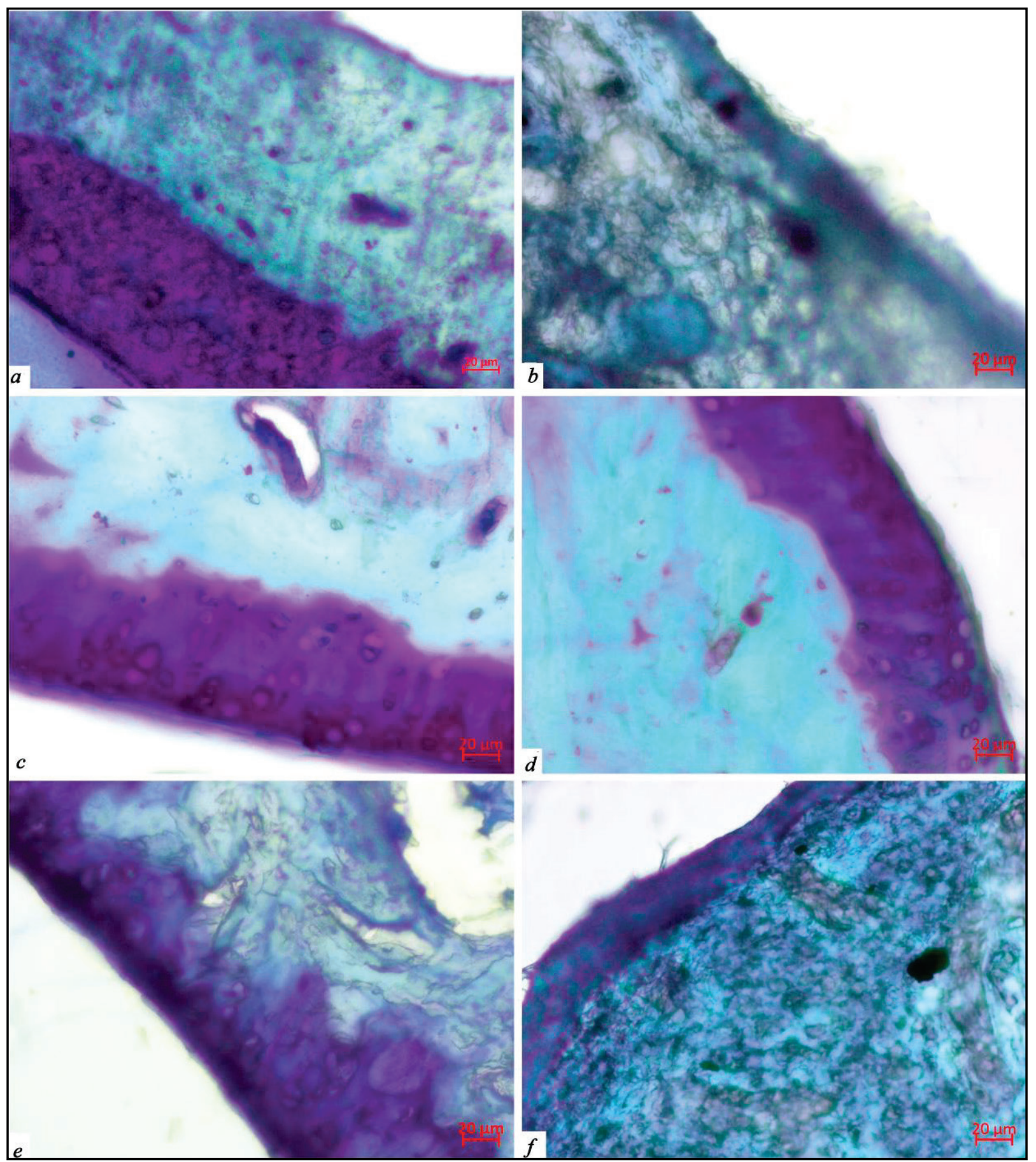

Fig. 4. Determination of glycosaminoglycans (purple) in the cartilage of animals with adjuvant arthritis and CrMMSC therapy (28 day): $a$-intact; $b$ - control (saline administration); $c$ - group 1 (local adiposed-derived CrMMSC administration); $d$ - group 2 (local cartilage-derived CrMMSC administration); $e$ - group 3 (generalized adiposed-derived CrMMSC administration); $f$-group 4 (generalized cartilage-derived CrMMSC administration); micrographs, light microscopy, toluidine blue staining 
From the above it follows that an increase in the relative area of the sites of staining to glycosaminoglycans in the articular cartilage was observed in animals treated with CrMMSCs. However, the severity of this process varied depending on the method of administration. A more pronounced process of restoring of glycosaminoglycan synthesis in the articular cartilage was observed under condition of local administration, regardless of the source of CrMMSCs.

The next stage of the study was to evaluate the content of COX-2 positive cells in the articular cartilage of animals with adjuvant arthritis and therapy with CrMMSC from adipose and cartilage tissues under local and generalized administration (Fig. 5). COX-2 is known to be expressed by macrophages, synoviocytes, fibroblasts, vascular smooth muscle, chondrocytes and endothelial cells under conditions of their induction by cyto- kines or growth factors. It is likely that prostaglandins formed under the action of COX-2 can directly or indirectly enhance the production of the enzyme itself by a positive feedback mechanism. A similar association was found in the retina in a model of concanavalin-induced inflammation in rats. Inhibition of COX-2 is considered as one of the main mechanisms of anti-inflammatory activity, because the selective inhibition of this cyclooxygenase can minimize many of the side effects observed with inhibition of cyclooxygenase 1 . COX-1 and COX-2 have almost the same molecular weight (70 and $72 \mathrm{kDa}$, respectively), almost identical amino acid sequences and almost completely identical catalytic sites. The results obtained in our study are shown that the relative area of COX-2 positively stained sites in the articular cartilage of intact animals was $7.9 \pm 0.7 \%$.

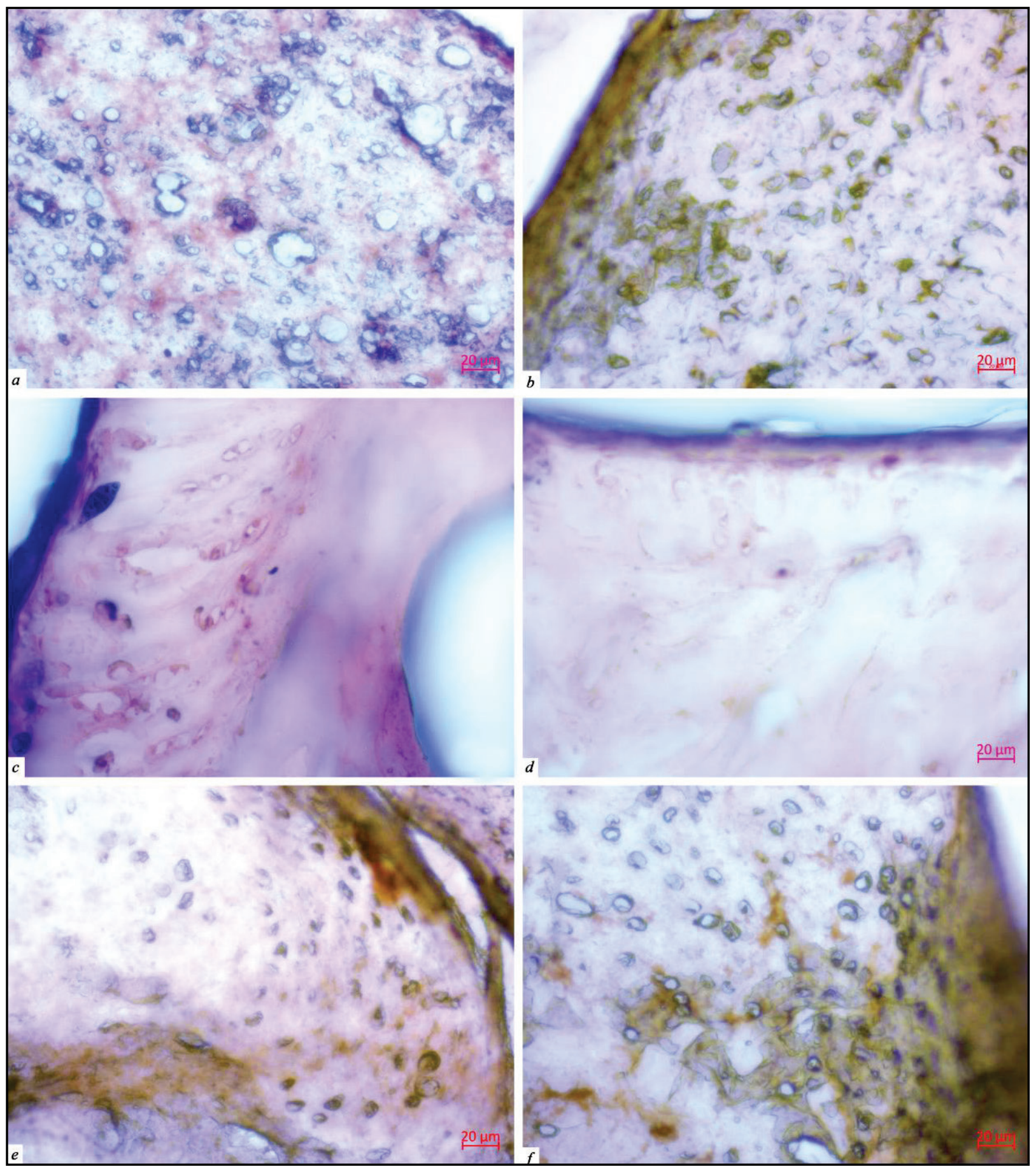

Fig. 5. Determination of COX-2 (brown) in the cartilage of animals with adjuvant arthritis

and CrMMSC therapy (28 day): $a$ - intact; $b$ - control (saline administration); $c$ - group 1 (local adiposed-derived CrMMSC administration);

$d$ - group 2 (local cartilage-derived CrMMSC administration); $e$ - group 3 (generalized adiposed-derived CrMMSC administration);

$f$-group 4 (generalized cartilage-derived CrMMSC administration); micrographs, light microscopy, immunohistochemical staining 
In the control group of animals with saline administration, the studied parameter was $56.3 \pm 2.8 \%$, which was 7.13-fold higher than in intact group. In animals with local administration of CrMMSCs from adipose and cartilage tissues, the relative area of COX-2 positively stained sites was $21.7 \pm 5.1 \%$ and $29.4 \pm 6.5 \%$ respectively, which was 2.59 - and 1.91 fold lower than the corresponding values in the control group of animals. In rats with generalized administration (groups 3 and 4), the studied index was $34.7 \pm 5.6 \%$ and $35.1 \pm 5.8 \%$ respectively, which was 1.62 - and 1.60 fold lower than the corresponding value in the control. However, it should be noted that in all studied groups, the COX-2 did not reach normal levels. Decreases in COX-2 content in articular cartilage were observed in animals treated with CrMMSCs. However, the severity of this process varied depending on the method of administration. A more pronounced process of reducing inflammation in the cartilaginous tissue was observed under local administration.

\section{Discussion}

According to the authors (Fu et al., 2017; Volkova et al., 2019; Jimenez-Puerta et al., 2020) MMSCs can promote regeneration not only by direct cell differentiation but also by secretion of biologically active substances known as growth factors. The latter are one of the most important molecular families involved in regeneration. With the help of protein receptors, growth factors bind to target cells. Receptor connections cause intracellular reactions, which, however, are not fully understood. It should be noted that the regulators of prochondrogenic cells and direct chondrogenic differentiation are a number of intracellular signaling molecules and growth factors, which include fibroblast growth factor (FGF), bone morphogenetic protein (BMP), platelet-derived growth factor (PDGF), insulin-like growth factor (IGF), epidermal factor (Jafri et al., 2020; Qasim etal., 2020).

Histological, histochemical and biochemical studies of the effectiveness of local and generalized administration of CrMMSCs from adipose and cartilage tissues in the correction of the acute phase of adjuvant arthritis showed that the animals of the control group with saline administration developed an inflammatory process throughout the observation period, which manifested in spread of chondrocyte-free zones, weakening of staining, loss of clarity of cartilage contours, reduced glycosaminoglycan content as well as decreased antioxidant system activity in serum and increased relative area of COX-2 positively stained sites in cartilage. At the same time, the local and generalized administration of CrMMSCs at the acute phase of adjuvant arthritis contributed to the normalization of the structural and functional tissue organization and had a tendency to reduce inflammation with complete recovery of blood parameters, which is consistent with previously published works on ability of CrMMSCs to influence the intensity of regenerative processes in cartilage tissue. These data are consistent with the results obtained on the use of native MMSCs (Kangari et al., 2020; Li et al., 2020), which led the researchers to recommend the creation of cryobanks of cells of stromal origin. This study also indicates that the changes that occurred in the cartilage tissue under the conditions of local administration of CrMMSCs from adipose and cartilage tissues covered unrestricted areas of the degenerative process and had a more pronounced regenerative effect compared to generalized method. Comparative evaluation of the use of CrMMSCs from adipose and cartilage tissues showed the absence of probable changes in the studied indicators under both local and generalized administration.

It is now known that MMSCs, as cells with a high level of CXR4 receptor expression to SDF-1 (stromal cell derived factor), are characterized by tropism to areas of tissue damage, where the content of SDF-1 increases (Bhakta et al., 2006; Fu et al., 2019). The results of generalized administration of CrMMSCs presented in this work confirm the hypothesis that stem cells are not only passively distributed throughout the body by the blood flow, but carry out targeted migration in the area of tissue damage. In addition, the corrective effect of generalized administration of CrMMSCs from the studied sources on the course of experimental adjuvant arthritis may be also related to their immunomodulatory properties. According to the authors (Kim et al., 2016; Wassmer \& Berishvili, 2020) to activate the immunosuppressive function of MMSCs in the body the mechanisms of action of IFN- $\gamma$ are triggered separately or together with
TNF $\alpha$, IL-1 $\alpha$ and IL-1 $1 \beta$. So, the obtained results showing the restoring effect of CrMMSCs on cartilage tissue allow us to draw a conclusion about the possibility of using the studied cell cultures in the treatment of rheumatoid arthritis under both local and generalized administration. This is a background for considering generalized administration as an alternative or supplement for local injection of cellular material to the joint. It is extremely important that the method of intravenous administration is a safe and non-traumatic procedure. Based on this provision, the systemic administration of MMSCs can be used in the treatment of this pathology. However, the question of the more distant effects of cell therapy remains open, in particular, the long duration of inhibition of the degenerativedystrophic process and the possible need for use of cells again.

\section{Conclusions}

Local and generalized administration of CrMMSCs from adipose and cartilage tissues led to a correction of the course of experimental adjuvant arthritis in rats: arthritis index, histostructure of damaged joint, activity of antioxidant defense system had a positive restorative dynamic. The use of a local method of cell administration compared to a generalized one had a more powerful effect on restoring the histostructure, content of glycosaminoglycans and COX-2 of cartilage in rats with adjuvant arthritis. These data can be used to substantiate and develop methods of arthritis treatment in clinical practice.

\section{References}

Bhakta, S., Hong, P., \& Koc, O. (2006). The surface adhesion molecule CXCR4 stimulates mesenchymal stem cell migration to stromal cell-derived factor-1 in vitro but does not decrease apoptosis under serum deprivation. Cardiovascular Revascularization Medicine, 7(1), 19-24.

Bullock, J., Rizvi, S., Saleh, A. M., Ahmed, S. S., Do, D. P., Ansari, R. A., \& Ahmed, J. (2018). Rheumatoid arthritis: A brief overview of the treatment. Medical Principles and Practice, 27(6), 501-507.

Burmester, G. R., \& Pope, J. E. (2017). Novel treatment strategies in rheumatoid arthritis. Lancet, 389(10086), 2338-2348.

Chen, K., Bao, Z., Tang, P., Gong, W., Yoshimura, T., \& Wang, J. M. (2018). Chemokines in homeostasis and diseases. Cellular and Molecular Immunology, 15(4), 324-334.

Choudhary, N., Bhatt, L. K., \& Prabhavalkar, K. S. (2018). Experimental animal models for rheumatoid arthritis. Immunopharmacology and Immunotoxicology, 40(3), 193-200

Conigliaro, P., Triggianese, P., De Martino, E., Fonti, G. L., Chimenti, M. S., Sunzini, F., Viola, A., Canofari, C., \& Perricone, R. (2019). Challenges in the treatment of Rheumatoid Arthritis. Autoimmunity Reviews, 18(7), 706-713.

Dey, P. (2018). Basic and advanced laboratory techniques in histopathology and cytology. Springer, Singapore.

Fan, X. L., Zhang, Y., Li, X., \& Fu, Q. L. (2020). Mechanisms underlying the protective effects of mesenchymal stem cell-based therapy. Cellular and Molecular Life Sciences, 77(14), 2771-2794.

Fitzsimmons, R., Mazurek, M. S., Soos, A., \& Simmons, C. A. (2018). Mesenchymal stromal/stem cells in regenerative medicine and tissue engineering. Stem Cells International, 2018, 8031718

Freitag, J., Bates, D., Boyd, R., Shah, K., Barnard, A., Huguenin, L., \& Tenen, A. (2016). Mesenchymal stem cell therapy in the treatment of osteoarthritis: Reparative pathways, safety and efficacy - a review. BMC Musculoskeletal Disorders, 17,230

Freyria, A. M., \& Mallein-Gerin, F. (2012). Chondrocytes or adult stem cells for cartilage repair: The indisputable role of growth factors. Injury, 43(3), 259-265.

Fu, X., Liu, G., Halim, A., Ju, Y., Luo, Q., \& Song, A. G. (2019). Mesenchymal stem cell migration and tissue repair. Cells, 8(8), 784.

Fu, Y., Karbaat, L., Wu, L., Leijten, J., Both, S. K., \& Karperien, M. (2017). Trophic effects of mesenchymal stem cells in tissue regeneration. Tissue Engineering. Part B, Reviews, 23(6), 515-528.

Gerwin, N., Bendele, A. M., Glasson, S., \& Carlson, C. S. (2010). The OARSI histopathology initiative - recommendations for histological assessments of osteoarthritis in the rat. Osteoarthritis and Cartilage, 18(S3), S24-S34.

Goryachev, D. V., \& Telnykh, M. Y. (2018). Planirovanie registracionnoj programmy issledovanij preparatov bazisnoj protivovospalitel'noj terapii revmatoidnogo artrita [Planning of a clinical data registry for basic anti-inflammatory drugs for the treatment of rheumatoid arthritis]. The Bulletin of the Scientific Centre for Expert Evaluation of Medicinal Products, 8(4), 238-245 (in Russian).

Ingawale, D. K., \& Patel, S. S. (2018). Hecogenin exhibits anti-arthritic activity in rats through suppression of pro-inflammatory cytokines in Complete Fre- 
und's adjuvant-induced arthritis. Immunopharmacology and Immunotoxicology, 40(1), 59-71.

Jafri, M. A., Kalamegam, G., Abbas, M., Al-Kaff, M., Ahmed, F., Bakhashab, S., Rasool, M., Naseer, M. I., Sinnadurai, V., \& Pushparaj, P. N. (2020). Deciphering the association of cytokines, chemokines, and growth factors in chondrogenic differentiation of human bone marrow mesenchymal stem cells using an ex vivo osteochondral culture system. Frontiers in Cell and Developmental Bio$\log , 7,380$.

Jimenez-Puerta, G. J., Marchal, J. A., López-Ruiz, E., \& Gálvez-Martín, P. (2020). Role of mesenchymal stromal cells as therapeutic agents: Potential mechanisms of action and implications in their clinical use. Journal of Clinical Medicine, $9(2), 445$.

Kangari, P., Talaei-Khozani, T., Razeghian-Jahromi, I., \& Razmkhah, M. (2020). Mesenchymal stem cells: Amazing remedies for bone and cartilage defects. Stem Cell Research and Therapy, 11(1), 492.

Kim, Y. K., Na, K. S., Myint, A. M., \& Leonard, B. E. (2016). The role of pro-inflammatory cytokines in neuroinflammation, neurogenesis and the neuroendocrine system in major depression. Progress in Neuro-Psychopharmacology and Biological Psychiatry, 64, 277-284.

Le, H., Xu, W., Zhuang, X., Chang, F., Wang, Y., \& Ding, J. (2020). Mesenchymal stem cells for cartilage regeneration. Journal of Tissue Engineering, 11, 2041731420943839.

Levato, R., Webb, W. R., Otto, I. A., Mensinga, A., Zhang, Y., van Rijen, M., van Weeren, R., Khan, I. M., \& Malda, J. (2017). The bio in the ink: Cartilage regeneration with bioprintable hydrogels and articular cartilage-derived progenitor cells. Acta Biomaterialia, 61, 41-53.

Li, X., Wang, M., Jing, X., Guo, W., Hao, C., Zhang, Y., Gao, S., Chen, M., Zhang, Z., Zhang, X., Shen, S., Zhang, B., Xian, H., Wang, Z., Wang, Y., Sui, X., Wang, A., Peng, J., Lu, S., Liu, S., \& Guo, Q. (2018). Bone marrow- and adipose tissue-derived mesenchymal stem cells: Characterization, differentiation, and applications in cartilage tissue engineering. Critical Reviews in Eukaryotic Gene Expression, 28(4), 285-310.

Neybecker, P., Henrionnet, C., Pape, E., Grossin, L., Mainard, D., Galois, L., Loeuille, D., Gillet, P., \& Pinzano, A. (2020). Respective stemness and chondrogenic potential of mesenchymal stem cells isolated from human bone marrow, synovial membrane, and synovial fluid. Stem Cell Research and Therapy, 11(1), 316.
Peng, L., Jia, Z., Yin, X., Zhang, X., Liu, Y., Chen, P., Ma, K., \& Zhou, C. (2008). Comparative analysis of mesenchymal stem cells from bone marrow, cartilage, and adipose tissue. Stem Cells and Development, 17(4), 761-773.

Phull, A. R., Nasir, B., ul Haq, I., \& Kim, S. J. (2018). Oxidative stress, consequences and ROS mediated cellular signaling in rheumatoid arthritis. Chemico-Biological Interactions, 281, 121-136.

Qasim, M., Chae, D. S., \& Lee, N. Y. (2020). Bioengineering strategies for bone and cartilage tissue regeneration using growth factors and stem cells. Journal of Biomedical Materials Research, Part A, 108(3), 394411.

Rana, A. K., Li, Y., Dang, Q., \& Yang, F. (2018). Monocytes in rheumatoid arthritis: Circulating precursors of macrophages and osteoclasts and, their heterogeneity and plasticity role in RA pathogenesis. International Immunopharmacology, 65, 348-359.

Sasaki, A., Mizuno, M., Mochizuki, M., \& Sekiya, I. (2019). Mesenchymal stem cells for cartilage regeneration in dogs. World Journal of Stem Cells, 11(5), 254-269.

Savvidou, O., Milonaki, M., Goumenos, S., Flevas, D., Papagelopoulos, P., \& Moutsatsou, P. (2019). Glucocorticoid signaling and osteoarthritis. Molecular and Cellular Endocrinology, 480, 153-166.

To, K., \& Khan, W. (2019). Mesenchymal stem cell transplantation in rheumatoid arthritis. In: Pham, P. (Ed.). Stem cells in clinical applications. Springer, Cham.

Volkova, N. A., \& Goltsev, A. N. (2015). Cryopreservation effect on proliferation and differentiation potential of cultured chorion cells. Cryo Letters, 36(1), 25-29.

Volkova, N. A., Yukhta, M. S., \& Goltsev, A. N. (2016). Morphological and functional characteristics of cryopreserved multipotent mesenchymal stromal cells from bone marrow, adipose tissue and tendons. Cell and Organ Transplantology, 4(2), 200-205.

Volkova, N. A., Yukhta, M. S., \& Goltsev, A. N. (2019). Influence of growth factors on cryopreserved mesenchymal stromal cells. Fiziolohichnyi Zhumal, 65(2), 12-21 (in Ukrainian).

Wassmer, C. H., \& Berishvili, E. (2020). Immunomodulatory properties of amniotic membrane derivatives and their potential in regenerative medicine. Current Diabetes Reports, 20(8), 31.

Zhang, F., \& Ma, C. (2018). Comparison of the effectiveness on intra-articular and subcutaneous TNF inhibitor in rheumatoid arthritis patients. Clinical Rheumatology, 37(1), 199-204. 